\title{
Níveis de conhecimento esperados dos alunos: uma ferramenta na análise de questões utilizadas na avaliação de uma Unidade de Ensino Potencialmente Significativa (UEPS)
}

Knowledge levels expected by students: a tool in the analysis of questions used in the evaluation of a Potentially Meaningful Teaching Units (PMTU)

\author{
T. N. Ribeiro ${ }^{1 *}$; D. N. Souza ${ }^{2}$ \\ ${ }^{I}$ DFCI, Universidade Federal de Sergipe, CEP 49500-000, Itabaiana-Sergipe, Brasil \\ ${ }^{2}$ DFI, Universidade Federal de Sergipe, CEP 49100-000, São Cristóvão-Sergipe, Brasil
}

*tnribeiro@globo.com

(Recebido em 08 de março de 2016; aceito em 29 de outubro de 2016)

\begin{abstract}
Este artigo tem por objetivo identificar os resultados acerca da análise inicial e final de uma Unidade de Ensino Potencialmente Significativa (UEPS), discutindo quais as questões que podem proporcionar a identificação das concepções prévias e da evolução conceitual acerca dos níveis de conhecimento esperados dos alunos. O referencial teórico utilizado foi o da Teoria da Aprendizagem Significativa de David P. Ausubel e dos níveis de conhecimento esperados dos alunos, de Robert. A pesquisa foi do tipo qualitativa, e utilizamos como metodologia um teste inicial para análise inicial dos conhecimentos prévios dos alunos e um teste final para análise da sua evolução conceitual. Concluímos que os Níveis de Conhecimento se tornaram uma importante ferramenta de sala de aula para construção e avaliação de uma UEPS, proporcionando um melhor direcionamento para o trabalho das situações e atividades utilizadas, o reconhecimento dos conhecimentos prévios e a evolução conceitual dos alunos.

Palavras-chave: Aprendizagem Significativa, Níveis de Conhecimento, Educação Matemática, Razões Trigonométricas.
\end{abstract}

This article aims to identify the results on the initial analysis and final analysis a Potentially Meaningful Teaching Units (PMTU), discussing what issues can provide the identification of previous conceptions and conceptual evolution of about the knowledge levels expected by students. The theoretical reference used was the theory Meaningful Learning of David P. Ausubel and knowledge levels expected by the students of Robert. The research was qualitative type and used as an initial test methodology for preview analysis of the students' prior knowledge and a final test to analysis of it conceptual evolution. We conclude that knowledge levels have become an important classroom tool for construction and evaluation of a PMTU, providing better guidance to the work of the used situations and activities, the recognition of preview knowledge and the conceptual evolution of the students.

Keywords: Meaningful Learning, Knowledge Levels, Education Mathematics, Ratios Trigonometric.

\section{INTRODUÇÃO}

Neste artigo investigamos o desenvolvimento da aprendizagem de alunos em situações de ensino desenvolvidas em uma Unidade de Ensino Potencialmente Significativa (UEPS) sobre conceitos referentes ao conteúdo razões trigonométricas no triângulo retângulo, a partir de situações aplicadas à Física. Foram investigadas as dificuldades encontradas pelos alunos quanto à mobilização dos conceitos e ferramentas matemáticas nas análises realizadas para identificação dos conhecimentos prévios dos alunos e na evolução conceitual desses.

Partindo da hipótese que a simples disponibilidade da UEPS, a partir de situações aplicadas à Física, pode incentivar a curiosidade e a imaginação dos estudantes, neste trabalho procurou-se discutir um modelo de ensino e aprendizagem que atenda às novas demandas da sociedade do conhecimento, levando em consideração o aprendiz.

Tendo como base a nossa prática docente, faz-se necessário pensar numa escola a partir dos seus próprios desafios, ligados à busca por uma melhor qualidade do processo de ensino e aprendizagem, objetivando a melhor formação do aluno, para que os conteúdos ensinados possam trazer 
conhecimentos significativos, estando inseridos nos frutos do saber contemporâneo de forma mais ativa, integradora e crítica.

Razões trigonométricas no triângulo retângulo é um conteúdo da Matemática que é abrangente, principalmente quando nos referimos a aplicações práticas nas várias áreas de atividades do ser humano, levando-se em consideração podem enriquecer a ação do professor em sala de aula. Em Física, por exemplo, são inúmeros os temas que se relacionam com razões trigonométricas no triângulo retângulo. Assim, no desenvolvimento desse conteúdo matemático podemos destacar a presença de temas como: lançamento de projéteis, vetores, plano inclinado, movimento circular, ondas, corrente alternada, entre outros. A articulação desses sinaliza tratamentos metodológicos e de interdisciplinarização de conteúdos educacionais como possibilidade de organização do trabalho pedagógico, que permite uma apreensão dos saberes, não mais marcada pela fragmentação, mas pela inter-relação entre conhecimentos diversos.

Para isso, acreditamos na emergência de materiais didáticos que possam buscar significados nas situações de aprendizagem. A nossa percepção é da necessidade de uma quase imediata ou instantânea construção de unidades de ensino que representem ambientes de aprendizagem potencialmente significativos para os alunos. Para isso, faz-se necessário adotar mecanismos de construção dessas unidades em sala de aula, aproveitando os conhecimentos prévios relevantes na estrutura cognitiva dos alunos, de forma a interagir com o próprio material construído, utilizandose de fatores idiossincráticos cognitivos e afetivos dos alunos.

As nossas inquietudes nos levaram aos seguintes questionamentos: Como evolui a aprendizagem de alunos em situações de ensino desenvolvidas em uma Unidade de Ensino Potencialmente Significativa (UEPS) sobre conceitos referentes ao conteúdo razões trigonométricas no triângulo retângulo, a partir de situações aplicadas à Física? Quais seriam as questões utilizadas para mobilizar os conhecimentos esperados pelos alunos na aplicação da UEPS?

Para responder a esses questionamentos, optamos por escolher como conteúdo as razões trigonométricas no triângulo retângulo, utilizando os princípios da UEPS em situações aplicadas à Física, numa abordagem do tipo qualitativa. Consideramos que essa abordagem nos forneceria uma relação direta entre a teoria e a prática educacional, de forma integrada com a realidade da sala de aula em situações de ensino. Para isso, foram escolhidas três turmas, compostas por um total de 43 alunos, dos quais: 16 alunos eram do curso de "Nivelamento" em Física, Química e Matemática do campus Prof. Alberto Carvalho da Universidade Federal de Sergipe (Turma 1); 13 alunos do Programa Institucional de Bolsas de Iniciação à Docência (PIBID) da mesma universidade (Turma 2); e 14 alunos da $3^{\circ}$ série do ensino médio (Turma 3) de uma escola pública de Aracaju, SE.

Para validar a pesquisa utilizamos como referencial teórico a Teoria da Aprendizagem Significativa, de David P. Ausubel [1,2]. A Teoria da Aprendizagem Significativa (TAS) tem como princípio o fato de que novas ideias expressas de forma simbólica se relacionam com aquilo que o aprendiz já sabe de forma não arbitrária e não literal, e que o produto desta interação ativa e integradora resulta no surgimento de um novo significado, que reflete a natureza substantiva e denotativa deste produto interativo [2].

Embora o princípio da TAS pareça simples, alguns pressupostos relevantes foram apresentados por Novak [3] no que diz respeito à determinação do que o aluno já sabe. Para o autor significa identificar os elementos relevantes ao que esperamos ensinar, ou identificar os conceitos de subsunções relevantes já existentes na estrutura cognitiva do aluno [3]. Por isso,

...à medida que nova experiência é adquirida e novo conhecimento é relacionado
a conceitos já existentes na mente das pessoas, estes conceitos tornam-se
elaborados ou modificados e, por isto, podem ser relacionados a um conjunto
mais amplo de novas informações em uma aprendizagem subsequente [3].

Isso significa que a TAS é uma teoria que reflete um processo cognitivo significativo e ativo da aprendizagem, no qual o novo conhecimento age no conhecimento especificamente relevante da estrutura cognitiva do aluno. Portanto, a construção do conhecimento na aprendizagem significativa se dá a partir das relações do novo conhecimento com o conhecimento prévio que os aprendizes adquirem ao decorrer da vida, com suas experiências vividas desde a infância. Em suma, o conhecimento prévio que o aluno possui, unido com aspectos afetivos, emotivos e o desejo de aprender, são aspectos imprescindíveis na construção do novo conhecimento desse sujeito. 
Para Ausubel [2], o tipo e o grau de significação desse conhecimento dependem de um processo integrador entre o material de instrução e as ideias relevantes existentes na estrutura cognitiva do aprendiz, que dependem de fatores como:

(1) as relações particulares hierárquicas e substantivas entre as ideias novas e as existentes (ancoradas) no processo de interação; (2) o grau de relevância particular das ideias ancoradas na estrutura cognitiva do aprendiz para com as novas ideias no material de instrução com as quais estão relacionadas; (3) o fato de um novo material de instrução estar ou não relacionado com ideias ancoradas relativamente específicas (particulares) no processo de aprendizagem significativa ou a conhecimentos anteriores mais gerais e difusos no armazém de memória relevante do aprendiz; e (4) variáveis da estrutura cognitiva tais como disponibilidade, estabilidade, longevidade e clareza das ideias ancoradas e respectiva capacidade de discriminação quer de ideias novas do material de aprendizagem, quer de ideias ancoradas relevantes na estrutura cognitiva [2].

Essa interação entre os conhecimentos prévios e o novo conhecimento se faz de modo não arbitrário e não literal. Na forma não arbitrária, o próprio material relaciona as ideias relevantes da estrutura cognitiva do aluno com o novo conhecimento. Na forma não literal, a tarefa de aprendizagem pode se relacionar com símbolos ou grupos de símbolos equivalentes, em termos ideários, à estrutura cognitiva do aluno, sem alterar o significado de forma significativa [2]. Ou seja, a nova informação tende a interagir com uma estrutura específica do conhecimento do aluno.

Para validar as questões da análise prévia e final utilizadas na UEPS e identificar a evolução conceitual dos alunos durante a pesquisa, utilizamos como referencial teórico a abordagem teórica de Robert [4] sobre os níveis de conhecimento esperados pelos alunos. Isso porque acreditamos que quando um aluno não consegue resolver determinado problema de Física, as dificuldades enfrentadas por ele não são somente matemáticas, mas também de interpretação do enunciado e de conteúdo, que nem sempre estão disponíveis na estrutura cognitiva do mesmo.

Robert [4] apresenta algumas ferramentas de análise epistemológica e didática dos conhecimentos matemáticos que devem ser ensinados no ensino médio e na universidade, levandose em consideração o trabalho do educador em se adaptar a cada nível de especificidade da Matemática escolhida, e os pressupostos cognitivos e didáticos adotados que possibilitarão desenvolver o conteúdo específico e os tipos de atividades esperadas dos estudantes para o desenvolvimento do conteúdo [4]. Para a autora, trata-se de rotular um determinado campo do conhecimento matemático (campo conceitual) correspondente a uma organização que seja coerente a este mesmo campo ou a uma parte dele, caracterizada por objetos matemáticos apresentados de certa maneira, teoremas sobre esses objetos, métodos associados a estes teoremas e problemas que os alunos podem resolver utilizando o nível de interesse ou método escolhido [4]. A autora classifica os níveis de conhecimento em: Técnico, Mobilizável e Disponível.

O Nível Técnico pode ser compreendido como um conjunto de operações isoladas, locais, corretas - diz respeito a uma ferramenta (incluindo definições). Refere-se às questões de uma forma direta e imediata, sendo mais relacionado com o funcionamento das questões do que com a sua compreensão [4]. No caso da nossa pesquisa, utilizamos questões de Nível Técnico somente em situações específicas no desenvolvimento das atividades da UEPS, para identificação e/ou aplicação das fórmulas das razões trigonométricas e do Teorema de Pitágoras, de forma explícita e sem a necessidade da mobilização do conteúdo, com o intuito de identificar se os alunos as reconheciam.

O Nível Mobilizável é mais amplo, porque compreende uma justaposição dos princípios de conhecimento em determinado campo ou domínio [4]. Neste nível, as atividades se apresentam de uma forma mais abrangente que no nível técnico, não sendo a resolução delas somente uma simples aplicação de um teorema, definição, conceito ou fórmula, uma vez que, como cita a Robert [4], acontece uma justaposição de saberes de certo domínio, oportunizando que vários métodos possam ser utilizados. A interação entre as razões trigonométricas no triângulo retângulo e situações da Física geraram questões do tipo mobilizável, uma vez que se fazia necessário a justaposição do conhecimento físico ao matemático.

No Nível Disponível, a tarefa deve requerer que o aluno seja capaz de resolver o que é proposto, sem indicações de roteiros para a obtenção do resultado. Para a solução, por exemplo, o aluno pode 
fornecer contraexemplos (como encontrar ou inventar), mudar de quadros, ou ainda aplicar métodos não previstos [4]. Ou seja, neste nível são propostas questões nas quais seja necessário provocar os alunos de forma a mobilizar os conhecimentos aprendidos e estimular a busca da estratégia mais adequada para as resoluções.

Aqui podemos exemplificar uma questão do Nível Disponível com uma situação na qual utilizamos o plano inclinado sem atrito para que os alunos identificassem as forças e as respectivas componentes que agem sobre o corpo e a aceleração ao longo do plano decorrente do ângulo de inclinação. Este exemplo teve por objetivo identificar os conhecimentos que os alunos tinham em relação à utilização das razões trigonométricas, porém era necessário também o conhecimento sobre a decomposição de vetores, grandezas, unidades de medida e proporcionalidade. Logo, cada aluno deveria recorrer a conhecimentos anteriores, inclusive de Física para a solução das questões.

Uma sequência de ensino fundamentada na Teoria da Aprendizagem Significativa tem por finalidade relacionar e fazer interagir de forma substantiva e não arbitrária os novos conhecimentos com o conhecimento prévio existente na estrutura cognitiva do aluno. Assim, no desenvolvimento de uma UEPS, busca-se um material de ensino não aleatório, plausível e sensível ao conhecimento prévio do aluno, de forma a oportunizar situações nas quais ele seja capaz de ampliar o conhecimento. Por isso, a sequência deve estar inserida em um processo de ensino ativo, pois, com ela, como expõe Ausubel, pretende-se oportunizar no mínimo:

(1) uma análise cognitiva necessária para se averiguar quais são os aspectos da estrutura cognitiva existente mais relevantes para o novo material potencialmente significativo; (2) algum grau de reconciliação com as ideias existentes na estrutura cognitiva, ou seja, apreensão de semelhanças e de diferenças e resolução de contradições reais ou aparentes entre conceitos e proposições novos e já enraizados; (3) reformulação do material de aprendizagem em termos dos antecedentes intelectuais idiossincráticos e do vocabulário do aprendiz em particular [2].

Para que a UEPS elaborada tenha o potencial de ser inserida nos princípios da Teoria da Aprendizagem Significativa, necessita ser estruturada nos fundamentos da diferenciação progressiva e da reconciliação integrativa, de forma a proporcionar aprendizagem, a retenção e a organização do tema proposto na estrutura cognitiva do aluno.

Dessa forma, para que a sequência didática seja classificada como uma unidade de ensino potencialmente significativa, Ausubel apresenta algumas condições:

(1) a capacidade de relação não arbitrária e não literal para com ideias particulares relevantes na estrutura cognitiva do aprendiz; (2) a capacidade de relação com a estrutura cognitiva particular de um aprendiz em particular - é mais propriamente uma característica do aprendiz do que o próprio material [2].

A partir dessas considerações, no decurso da aprendizagem com UEPS, o aluno deve relacionar os novos conceitos e proposições com a própria estrutura cognitiva idiossincrática. Para que isso ocorra é necessário que o material apresente alguns aspectos importantes: ele deve ser diversificado em termos de estratégias e ferramentas de ensino; prever atividades diversificadas sobre um mesmo conceito, para atacar as semelhanças e diferenças que possam confundir as novas ideias adquiridas com as já existentes na estrutura cognitiva dos aprendizes; apresentar clareza e estabilidade nos subsunçores utilizados; buscar desenvolver a aprendizagem a partir da motivação de questionamentos, evitando respostas prontas durante a evolução dos temas, estimulando o diálogo e a criticidade em sala de aula; incentivar que os próprios alunos construam situações-problema nas unidades; privilegiar atividades colaborativas entre os alunos; e possibilitar avaliações sistemáticas.

Aqui descrevemos os resultados acerca da análise inicial e final, e discutimos quais as questões que podem proporcionar a identificação dos conhecimentos prévios dos alunos e a evolução conceitual deles, considerando os níveis de conhecimento esperados.

O questionário utilizado para análise inicial teve como objetivo identificar, a partir de situaçõesproblema, os conhecimentos prévios dos alunos e os conteúdos de Física que poderiam gerar um conjunto de situações que servissem de aporte para o desenvolvimento de uma UEPS para o tema razões trigonométricas no triângulo retângulo. Essa análise fez-se necessária porque a partir dela pôde-se identificar os subsunçores necessários para a aprendizagem significativa. O teste de análise 
final foi aplicado, também a partir de situações-problema, com a finalidade de identificar a evolução conceitual dos alunos e concluir se a UEPS proposta foi potencialmente significativa. Os resultados do teste permitiram destacar os pontos positivos e negativos da utilização da mesma.

\section{MATERIAIS E MÉTODOS}

Tendo por base que esta pesquisa teve por objetivo investigar os conhecimentos prévios e a evolução conceitual dos alunos sobre o conteúdo razões trigonométricas no triângulo retângulo utilizando os princípios das Unidades de Ensino Potencialmente Significativas em situações aplicadas à Física, procuramos também analisar as estratégias e procedimentos de ensino que norteiam uma aprendizagem significativa de forma a investigar o desenvolvimento da aprendizagem visando à identificação da evolução cognitiva dos alunos; para isso, utilizamos para a coleta dos dados, numa abordagem qualitativa, os seguintes instrumentos: análise prévia, atividades na UEPS e análise final.

A análise inicial foi realizada a partir de um teste inicial composto por seis questões de nível mobilizável e uma de nível disponível. Ele foi construído acerca dos seguintes temas: conceituação e caracterização do triângulo retângulo, Teorema de Pitágoras, semelhança de triângulos e razões trigonométricas.

$\mathrm{Na}$ fase de experimentação, foram aplicadas as atividades da UEPS durante quatro encontros presenciais, totalizando oito horas/aula, nos quais, professor/pesquisador e alunos desenvolveram as atividades propostas visando identificar a potencialidade da UEPS. A unidade foi elaborada considerando-se os aspectos sequenciais estabelecidos por Moreira [5], definidos a partir do tema razões trigonométricas no triângulo, tendo como situações-problema questões da Física, abordando sobre plano inclinado, princípio de propagação retilínea da luz, deslocamento vetorial e componentes vetoriais da Força.

As atividades foram desenvolvidas por meio de estratégias de ensino diversificadas, partindo de situações-problema, na qual apresentávamos o conhecimento a ser ensinado/aprendido sobre o tema, levando em consideração a diferenciação progressiva, isto é, começando com aspectos mais gerais e inclusivos sobre o tema e em seguida abordando aspectos mais gerais e com maior complexidade em relação à situação inicial, de forma a possibilitar a reconciliação integradora. As estratégias utilizadas foram: o software GeoGebra para a conceitualização do triângulo retângulo, vídeo documentário e questões-problema envolvendo o Teorema de Pitágoras, software de animação e experimento para as razões trigonométricas.

Em seguida, com o objetivo de identificar a evolução conceitual dos alunos após aplicação da UEPS, foi realizada uma análise final a partir de um teste final, que teve por finalidade caracterizar a evolução conceitual dos alunos e observar se a UEPS proposta foi potencialmente significativa, além de destacar os pontos positivos e negativos da utilização da mesma. O teste, que foi composto por cinco questões de nível de conhecimento mobilizável e duas do nível disponível, tratou dos mesmos temas presentes no teste inicial para análise prévia, porém com situações mais complexas, visando investigar a eficiência da UEPS no processo de ensino e aprendizagem.

\section{RESULTADOS E DISCUSSÃO}

A partir da análise prévia observamos um bom índice de acerto para a conceituação e caracterização do triângulo retângulo, mas nos outros temas o rendimento não foi o esperado, considerando que os alunos participantes já tinham tido aulas sobre o tema na educação básica. Aqui podemos nos questionar, mesmo já tendo sido abordado o conteúdo na educação básica, será que esses alunos o estudaram adequadamente?

Identificamos nas respostas do teste inicial que os alunos mobilizavam conceituações referentes à Física e não ao conteúdo matemático, o que demonstra, como previsto por Ausubel [2], que os discursos dos alunos expressam experiências de aprendizagem passadas, que influenciam, de maneira positiva ou negativa, a nova aprendizagem, acarretando em uma aprendizagem nova, que possivelmente pode ser afetada pelos conhecimentos prévios existentes nas estruturas cognitivas dos alunos. 
A única questão de nível disponível, que tratava sobre a formação da sombra de uma edificação a partir dos raios de Sol, que eram representados com uma inclinação de $60^{\circ}$ em relação ao plano horizontal, teve o menor índice de acerto de todas as questões do teste inicial (apenas 13,9\%). Evidenciando o grau de desenvolvimento dos conhecimentos dos alunos sobre o nível de conhecimento exigido.

Verificamos, assim, que no teste inicial os alunos conseguiram resolver as questões nas quais necessitavam da mobilização de conhecimentos matemáticos explícitos e não conseguiram resolver ou tiveram dificuldades no entendimento daquelas questões com indicações não explícitas sobre o que seria mais adequado para respondê-las.

A partir do teste de análise final identificamos que as respostas da questão 01 (quadro 01), que trata da identificação das razões trigonométricas no triângulo retângulo, o índice de acerto observado foi de $83,7 \%$, enquanto que em uma das atividades da UEPS, que era de nível de conhecimento técnico, evidenciamos que todos os alunos conseguiram identificar tais razões. Assim, identificamos dificuldade na resolução de questão por ser do nível mobilizável, uma vez que a interpretação do quesito não pode ser realizada de forma direta e imediata, como se espera de questão de nível de conhecimento técnico.

QUADRO 01 - Questão 01 do teste final

01 - A partir do triângulo retângulo abaixo e fixando o ângulo $\beta$, efetue a correspondência:

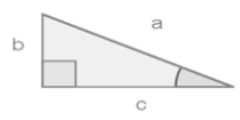
(1) $\operatorname{sen} \beta$
(2) $\cos \beta$
(3) $\operatorname{tg} \beta$
(4) $\operatorname{cotg} \beta$

$\begin{array}{ll}( & ) \mathrm{c} / \mathrm{a} \\ ( & ) \mathrm{c} / \mathrm{b} \\ ( & ) \mathrm{b} / \mathrm{c} \\ ( & ) \mathrm{b} / \mathrm{a}\end{array}$

FONTE: $\mathrm{O}$ autor (2015)

Nas questões 02 e 03 (quadro 02), tivemos por objetivo aplicar as razões trigonométricas no triângulo retângulo a partir de situações da Física; as questões são de nível mobilizável e disponível, respectivamente. $\mathrm{O}$ índice de acerto para a questão 02 foi de $74,4 \%$ e para a questão 03 de $67,4 \%$, enquanto na questão do teste inicial, que tratava do mesmo tema, esse índice foi de 13,9\%. A melhoria dos índices de acerto demonstra uma evolução conceitual, que poderá estar associada à potencialidade da UEPS e ao papel mediador do professor na busca de conferir significados às situações-problema. Afinal, são essas situações que dão sentido aos novos conhecimentos, além de funcionarem como organizadores prévios. Porém, ainda identificamos dificuldades de interpretação e representação de questões em nível mobilizável e disponível, inerentes de alunos que têm como hábito resolver questões de nível técnico, devido à forma de aquisição de conhecimento centrado na aprendizagem mecânica.

QUADRO 02 - Questões 02 e 03 do teste final

02 - (COVESP-PE) Um barco atravessa um rio num trecho onde a largura é $100 \mathrm{~m}$, seguindo uma direção que forma um ângulo de $30^{\circ} \mathrm{com}$ uma das margens. Assinale a alternativa certa para a distância percorrida pelo barco para atravessar o rio.
a) $100 \mathrm{~m}$
b) $200 \mathrm{~m}$
c) $150 \mathrm{~m}$
d) $250 \mathrm{~m}$

03 - (UNIFOR-CE) Em certa hora do dia, os raios do Sol incidem sobre um local plano com uma inclinação de $60^{\circ}$ em relação à horizontal. Nesse momento, o comprimento da sombra de uma construção de $6 \mathrm{~m}$ de altura será, aproximadamente:

a) $10,2 \mathrm{~m} \quad$ b) $8,5 \mathrm{~m}$ c) $5,9 \mathrm{~m}$ d) $4,2 \mathrm{~m}$ e) $3,4 \mathrm{~m}$ 
As questões 04 e 05 (quadro 03) são de níveis de conhecimento mobilizável e tinham como objetivo identificar a aprendizagem conceitual do Teorema de Pitágoras. O índice de acerto para a questão 04 foi de $67,4 \%$ e para a questão 05 foi de $86,0 \%$, enquanto na questão do teste inicial, que tratava do mesmo tema, esse índice foi de 46,5\%. Esses índices demonstram uma evolução conceitual; mas vale salientar que alguns alunos continuaram a ter dificuldades de interpretação e representação de questões em nível mobilizável. Mais uma vez demonstrando que a utilização de questões de nível técnico é tão frequente no cotidiano escolar dos alunos que eles não conseguem interpretar e mobilizar de forma satisfatória os conhecimentos matemáticos desenvolvidos durante a aplicação da UEPS.

\section{QUADRO 03 - Questões 04 e 05 do teste final}

04 - (MOJI-SP) Uma escada que mede $4 \mathrm{~m}$ tem uma de suas extremidades amparada no topo de um muro, e a outra extremidade dista $2,4 \mathrm{~m}$ da base do muro. A altura do muro é:
a) $2,3 \mathrm{~m}$
b) $3,0 \mathrm{~m}$
c) $3,2 \mathrm{~m}$
d) $3,8 \mathrm{~m}$

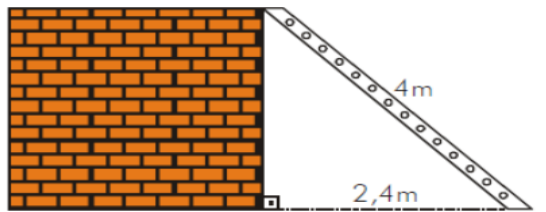

05 - Para determinar a distância de uma margem à outra do rio, um navegante mediu a distância de partida no ponto $\mathrm{C}$ até atracar no outro lado da margem no ponto $\mathrm{B}$ da margem do rio, encontrando $50 \mathrm{~m}$. Sabendo que a distância entre os pontos A e B é de $30 \mathrm{~m}$, determine a distância entre a margem $\mathrm{C}$ até $\mathrm{A}$.

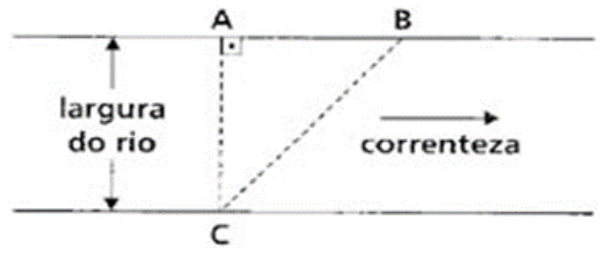

FONTE: MOJI-SP e AUTOR (2015)

Na questão 06 (quadro 04), de nível disponível, tivemos por objetivo a verificação da compreensão dos alunos acerca da aplicação de semelhança de triângulos a partir do princípio de propagação retilínea da luz. O índice de acerto foi de 86,0\%, enquanto na questão de atividade contida na UEPS, que tratava sobre o mesmo tema, foi de 72,0\%. Demonstrando mais uma vez evolução conceitual após o trabalho com a unidade de ensino.

QUADRO 04 - Questão 06 do teste final.

06 - Um menino de 1,5 m de altura produz uma sombra de $50 \mathrm{~cm}$. No mesmo instante, um prédio próximo do menino produz uma sombra de $20 \mathrm{~m}$. A altura do prédio, em metros, é:
(a) 20
(b) 30
(c) 50
(d) 60
(e) 10

FONTE: O autor (2015)

A questão 07 (quadro 05), de nível mobilizável, teve por objetivo verificar a evolução conceitual sobre a aplicação das razões trigonométricas no triângulo retângulo, tendo como situação o plano inclinado. O índice de acerto dos alunos foi de 90,7\%, demonstrando que eles permaneceram interpretando as situações adequadamente e que utilizaram corretamente as razões trigonométricas seno e cosseno. Na atividade contida na UEPS o índice médio de acerto de 89,9\%. 
QUADRO 05 - Questão 07 do teste final.

07 - Um corpo de peso $100 \mathrm{~N}$ é abandonado sobre um plano inclinado sem atrito, como mostra a figura. Sabendo que $\theta=60^{\circ}$, determine:

a) a intensidade da força normal exercida pelo plano inclinado sobre o bloco;

b) o módulo da aceleração adquirida pelo bloco;

c) a intensidade da força resultante exercida sobre o bloco.

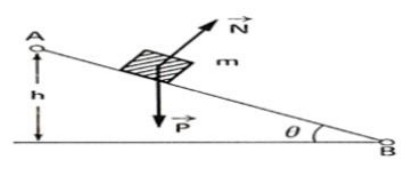

FONTE: O autor (2015)

A comparação dos resultados obtidos no teste final com os resultados do teste inicial sugere que a sequência de ensino da UEPS foi potencialmente significativa na construção dos conceitos, indicando uma evolução dos conhecimentos dos alunos de níveis mais concretos e com pouca abstração para níveis conceituais mais complexos.

\section{CONCLUSÃO}

Concluímos que estruturar as análises prévia e final da UEPS segundo os Níveis de Conhecimento foi eficaz na definição do grau de dificuldade das questões e para a análise da evolução conceitual dos alunos participantes da pesquisa, oportunizando a avaliação do material didático utilizado. Também nos permitiu uma análise de como os alunos aprenderam e articularam o conhecimento matemático a partir das situações da Física

Em relação ao tema razões trigonométricas no triângulo retângulo, a avaliação dos testes considerando os Níveis de Conhecimento permitiu demonstrar que a UEPS deve abordar, como ocorreu, o conteúdo de uma maneira mais profunda, uma vez que auxilia o desenvolvimento dos níveis mobilizável e disponível, essenciais para o trabalho que foi desempenhado nas atividades e na interação entre as disciplinas Física e Matemática.

Também identificamos, a partir dos dados coletados, que, após aplicação da UEPS, as dificuldades apresentadas pelos alunos não estavam relacionadas diretamente ao conteúdo razões trigonométricas no triângulo retângulo, uma vez que eles conseguiram identificar os conceitos trigonométricos em uma determinada situação, embora não tenham sido capazes de aplicá-los em situações-problema adequadamente. Isso caracteriza uma predominância de conhecimento de nível técnico evidente nos alunos participantes da pesquisa.

Tendo como base as considerações de Robert (4), podemos concluir que a identificação dos níveis de conhecimento nas questões se tornaram uma importante etapa no que diz respeito à construção e avaliação de uma UEPS, porque proporciona um melhor direcionamento para o trabalho das situações e atividades utilizadas, auxiliando no reconhecimento dos conhecimentos prévios e na avaliação da evolução conceitual dos alunos.

\section{REFERÊNCIAS BIBLIOGRÁFICAS}

1. Ausubel, DP. Psicologia educativa. Editorial Trillas, México, 1978. Tradução Roberto Helier Domínguez.

2. Ausubel, DP. Aquisição e retenção de conhecimentos: uma perspectiva cognitiva. Plátano edições técnicas, 2003, Lisboa/Portugal. Tradução de The acquisition and retention of knowledge: a cognitive view. Dordrecht: Kluwer Academic Publishers, 2000.

3. Novak, JD. Uma teoria de educação. Editora Pioneira, São Paulo, 1981.

4. Robert, A. Quelques outils d'analyse epistemologique et didactique de connaissances mathématiques à enseigner au lycée et à l'université. Actes de la IX école d'été de didactique des mathématiques. p.192212, Houlgate, França, 1997.

5. Moreira, MA. Unidades de Enseñanza Potencialmente Significativas - UEPS. Aprendizagem Significativa em Revista/Meaningful Learning Review - V1(2), pp. 43-63, 2011. 
6. Moreira, MA. Aprendizagem significativa: a teoria e textos complementares. Editora Livraria da Física, 2011, São Paulo/SP.

7. Novak, JD; Gowin, DB. Aprender a aprender. Lisboa, Plátano edições técnicas, 1984. Tradução Carla Valadares.

8. Ribeiro, TN. O ensino de razões trigonométricas no triângulo retângulo a partir de situações aplicadas à física: um estudo baseado nas Unidades de Ensino Potencialmente Significativas (UEPS). Tese de doutoramento, Universidade Anhanguera de São Paulo, 2015. 